\title{
Human platelets mediate iron release from transferrin by adenine nucleotide-dependent and -independent mechanisms
}

\author{
Joan K. Brieland ', Margaret C.M. Vissers ${ }^{2}$, Sem H. Phan ${ }^{2}$ and Joseph C. Fantone ${ }^{2}$ \\ 'Unit for Laboratory Animal Medicine and 2 Department of Pathology, The University of Michigan Medical School, \\ Ann Arbor, MI (U.S.A.)
}

(Received 9 March 1988)

(Revised manuscript received 4 October 1988)

Key words: Transferrin; Iron release; Platelet; Adenine nucleotide; (Human)

We assessed the ability of platelet sowiluates and mediators secreted by unstimulated and thrombin-stimulated platelets to facilitate the release of iron frosi transferrin. Platelet sonicates and platelet conditioned media potentiated the release of iron from transferrin. The rate of release of iron was dependent on the $\mathrm{pH}$ of the reaction and amount of platelet sample added. Conditioned media from thrombin-stimulated platelets was more effective in mediating the release of iron from transferrin than was conditioned media from unstimulated cells. The rate of iron released from transferrin following addition of ATP and ADP in amounts equivalent to that present in platelet conditioned media was significantly less than the rate of iron released following the addition of conditioned media from platelets. Depletion of ATP and ADP in platelet conditioned media by incubation with apyrase only partially inhibited their ability to enhance the rate of iron release from transferrin. These observations indicate that platelets enhance the release of iron from transferrin by adenine nucleotide-dependent and -independent mechanisms. These observations are consistent with the hypothesis that platelets promote oxidant-induced tissue injury at sights of inflammation secondary to their ability to enhance the local release of iron from transferrin.

\section{Introduction}

Free radical-mediated peroxidation of cell membranes plays an important role in the pathogenesis of several disease states [1]. Both the initiation and propagation of lipid peroxidation may be enhanced by the availability of iron in either its oxidized $\left(\mathrm{Fe}^{3+}\right)$ or reduced $\left(\mathrm{Fe}^{2+}\right)$ form. $\mathrm{Fe}^{2+}$ can promote hydroxyl radical production from hydrogen peroxide and both reduced and oxidized iron may function to propagate peroxidation reactions secondary to reaction with lipid hydroperoxides [1,2]. Iron $\left(\mathrm{Fe}^{3+}\right)$ is carried in the vertebrate bloodstream bound to transferrin, in a ternary complex involving transferrin, iron and bicarbonate [3-7] and is unable to effectively promuie lipid peroxidation in this bound state. Results of previous studies indicate that the adenine nucleotides, adenosine diphosphate (ADP) and adenosine triphosphate (ATP), can promote the release of iron from the iron-transferrin-bicarbonate complex by accelerating the exchange/ dissociation of transferrin-bound bicarbonate [8]. In the

Correspondence: J.K. Brieland, Box 0614 ULAM, Animal Research Facility, University of Michigan, Ann Arbor, MI 48109, U.S.A. absence of bicarbonate, there is no stable interaction between $\mathrm{Fe}^{3+}$ and transferrin which results in labilization of the iron [9].

Platelet aggregation and mediator secretion are essential for normal hemostasis $[10,11]$ and play an important ivle in the inflammatory response. Human platelets spontaneously secrete ADP and ATP and the secretion of these nucleotides is enhanced following platelet aggregation [10]. The effect of secretion of adenine nucleotides by platelets on the release of iron from transferrin has not been thoroughly investigated. Therefore, we evaluated the effect of the addition of platelet sonicates and conditioned media from unstimulated and thrombin-stimulated platelets on the rate of iron released from transferrin. The relative role of platelet-derived adenine nucleotides in mediating the release of iron from transferrin was determined.

\section{Materials and Methods}

All chemicals were obtained from Sigma Chemical Co. (St. Louis, MO) unless otherwise noted. Human fibrinogen was obtained from Kabi Diagnostica (Stockholm, Sweden). Human platelets were obtained from healthy volunteers. 


\section{Collection of human platelets}

Human platelets were harvested from citrated blood from healthy volunteers as previously described [12] with minor modifications. Platelet rich plasma, obtained by centrifugation of whole blood $(280 \times \mathrm{g}, 15 \mathrm{~min}$, $20^{\circ} \mathrm{C}$ ), was centrifuged $\left(200 \times \mathrm{g}, 10 \mathrm{~min}, 20^{\circ} \mathrm{C}\right)$ to remove any remaining leukocytes and erythrocytes. $1 \mathrm{ml}$ of citrate solution $(38 \mathrm{mM}$ citric acid, $75 \mathrm{mM}$ sodium citrate) was added for every $10 \mathrm{ml}$ platelet-rich plasma and the suspension was centrifuged $(2000 \times \mathrm{g}, 10 \mathrm{~min}$, $20^{\circ} \mathrm{C}$ ). The resultant platelet pellet was washed twice in salire to remove any remaining citrate and was resuspended at the $3.6 \cdot 10^{8}$ platelets $/ \mathrm{ml}$ in Hanks' balanced salt solution (HBSS).

\section{Preparation of human platelet sonicates}

Human platelets $\left(3.6 \cdot 10^{8}\right.$ platelets $\left./ \mathrm{ml}\right)$ were pulse sonicated for $30 \mathrm{~s}$ on ice using a microprobe dismembranator. Platelet sonicates were centrifuged $(2000 \times g$, $10 \mathrm{~min}, 20^{\circ} \mathrm{C}$ ) to remove any remaining intact platelets. The supernatant was collected and assayed as described below.

\section{Preparation of conditioned media from unstimulated and} thrombin-stimulated platelets

Human platelets $\left(3.6 \cdot 10^{8}\right.$ platelets $\left./ \mathrm{ml}\right)$ were preincubated with human fibrinogen at a final concentration of $0.1 \mathrm{mg} / \mathrm{ml}$ for $5 \mathrm{~min}$ in a $37^{\circ} \mathrm{C}$ shaking water bath. Following incubation with fibrinogen, the platelet suspension was divided into two fractions of equal platelet concentration. One of the fractions was stimulated with thrombin at a final concentration of $5 \mathrm{U} / \mathrm{ml}$. Both unstimulated and thrombin-stimulated platelets were incubated for an additional $5 \mathrm{~min}$ in a $37^{\circ} \mathrm{C}$ shaking water bath. The platelet suspensions were centrifuged $\left(2000 \times \mathrm{g}, 10 \mathrm{~min}, 20^{\circ} \mathrm{C}\right)$ to remove intact platelets. The conditioned media, containing mediators secreted from unstimulated and thrombin-stimulated platelets, was assayed as described below.

\section{Incubation of adenine nucleotides and platelet conditioned nedia with apyrase}

In select experiments ATP, ADP and conditioned media from unstimulated and thrombin-stimulated platelets were incubated with apyrase, an adenosine diphosphatase and triphosphatase, at a final concentration of $4.9 \mathrm{U} / \mathrm{ml}$ for $10 \mathrm{~min}$ in a $37^{\circ} \mathrm{C}$ shaking water bath prior to being assayed as described below.

\section{Measurement of iron release from transferrin}

The initial rate of iron released from transferrin was monitored spectrophotometrically $(295 \mathrm{~nm})$ on a Cary 210 dual beam spectrophotometer as described previously with minor modifications [7]. Buffer $(20 \mathrm{mM}$ Hepes for assays of $\mathrm{pH}>6.0,0.1 \mathrm{M}$ sodium acetate for assays of $\mathrm{pH}<6.0)$, iron saturated transferrin $(0.015$
$\mathrm{mM}$, final) and deferoxamine (2.5 $\mathrm{mM}$, final) were added to the sample curvette in a final volume of $1 \mathrm{ml}$. The basal rate of iron released from transferrin at $\mathrm{pH}$ 7.4 was determined spectrophotometrically by the initial change in absorbance at $295 \mathrm{~nm}$ (monitored over 1 min). In subsequent assays, ATP, platelet sonicates and conditioned media from thrombin-stimulated platelets were added to the reaction mixture in both curvettes and the initial rate of iron released from transferrin was calculated as described above. The release of $30 \mathrm{nmol}$ of iron corresponds to a change in absorbance of approx. 0.358 . The magnitude of the absorbance changes observed spectrophotometrically in the following experiments was from 0.012 to $\mathbf{0 . 0 7 0}$.

High pressure liquid chromatography analysis of adenine nucleotides in platelet sonicates and platelet conditioned media

Platelet sonicates and conditioned media from platelets were prepared as described above. The sonicates and conditioned media were concentrated in Centricon 10 microconcentrators (Amicon, Danrus, MA) by centrifugation $\left(2000 \times \mathrm{g}, 30 \mathrm{~min}, 4^{\circ} \mathrm{C}\right)$. Reversed phase high pressure liquid chromatography (HPLC) was used to separate nucleotides in the resultant platelet samples [13]. The chromatographic system included a Vista 5560 HPLC system (Varian Instruments, Palo Alto, CA) equipped with a UV-200 variable wavelength UV-visible detector, a Rheodyne Model 7126 automatic injector and a Model 402 data system. The solvent system consisted of $5 \mathrm{mM}$ tetrabutyl ammonium phosphate (pH 7.0) (A) and $5 \mathrm{mM}$ tetrabutyl ammonium phosphate and 30\% acetonitrile (pH 7.0) (B). The solvent flow rate was $1 \mathrm{ml} / \mathrm{min}$. Separation of nucleotides was accomplished using a $\mathrm{C}_{18}$ column (Supelco, Bellefonte, PA). Sample size was $50 \mu 1$ per injection and detection was at $254 \mathrm{~nm}$. Samples of platelet sonicate or conditioned media from unstimulated or thrombin-stimulated platelets were ultrafiltered through Centricon 10 microconcentrators and then injection into the column. Separation of AMP, ADP and ATP occurred with retention times of 15,26 , and $31 \mathrm{~min}$, respectively.

Characterization of the platelet-derived mediator(s) which facilitated the release of iron from transferrin

The molecular weight of the platelet-derived mediator(s) which facilitated the release of iron from transferrin was assessed. Conditioned media from thrombinstimulated platelets was centrifuged $(2000 \times \mathrm{g}, 20 \mathrm{~min}$, $4^{\circ} \mathrm{C}$ ) in Centricon 10 microconcentrators (molecular weight cutoff 10000). The ability of the filtrate to promote the release of iron from transferrin was assayed as described above. The effect of extreme temperatures on the activity of the platelet-derived mediator(s) was also assessed. Conditioned media from thrombinstimulated platelets was frozen $\left(-20^{\circ} \mathrm{C}, 24 \mathrm{~h}\right)$ or heated 
$\left(80^{\circ} \mathrm{C}, 30 \mathrm{~min}\right)$. The ability of this 'treated' conditioned media to facilitate the release of iron from transferrin was assayed as described above. Conditioned media from thrombin-stimulated platelets was also subjected to lipid extraction as described previously [14]. The ability of the lipid and aqueous fraction to facilitate the release of iron from transferrin was determined as described above.

\section{Statistical analysis}

All data represent the mean \pm the standard error (S.E.) from at least three experiments. Platelets used in a given experiment were from a single healthy donor, different donors were used for each experiment. The Student's paired $t$-test was used to evaluate differences between individual treatment groups and those differences with $P<0.05$ were deemed significant.

\section{Results}

\section{The effect of $\mathrm{pH}$ on the rate of iron released from transfer- rin}

The effect of $\mathrm{pH}$ on the initial rate of iron release from transferrin was determined. Consistent with the observations of others $[3,7,15,16]$, the rate of iron released from transferrin was enhanced as the hydrogen ion concentration was increased (Fig. 1). While the rate of iron released from transferrin spontaneously at $\mathrm{pH}>$ 6.2 was undetectable, the addition of ATP $(1.25 \mu \mathrm{M})$ or platelet sonicates $\left(9 \cdot 10^{6}\right.$ platelet equivalents) to transferrin at pH 6.2 facilitated a small increase in the rate of iron released from transferrin $(<1 \mathrm{nmol} / \mathrm{min})$. As the $\mathrm{pH}$ was decreased from 6.2 to 5.3 , the rate of iron released was increased both spontaneously and following the addition of ATP or platelet sonicates. All subse-

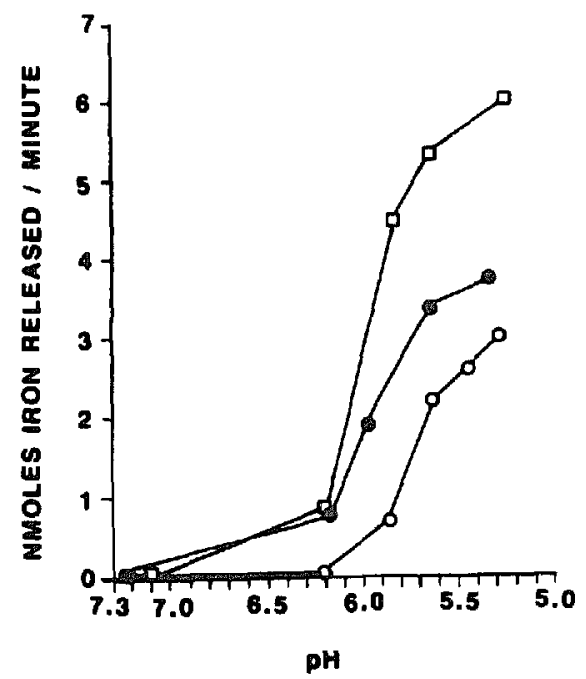

Fig. 1. Iron released ( $\mathrm{nmol} / \mathrm{min}$ ) from transferrin spontaneously and following the addition of ATP $(1.25 \mu \mathrm{M})$ and platelet sonicates $\left(9 \cdot 10^{6}\right.$ platelet equivalents) at $\mathrm{pH} 7.3$ to 5.3. $\bigcirc$, spontaneous release; 1 , ATP; $\square$, platelet sonicates.

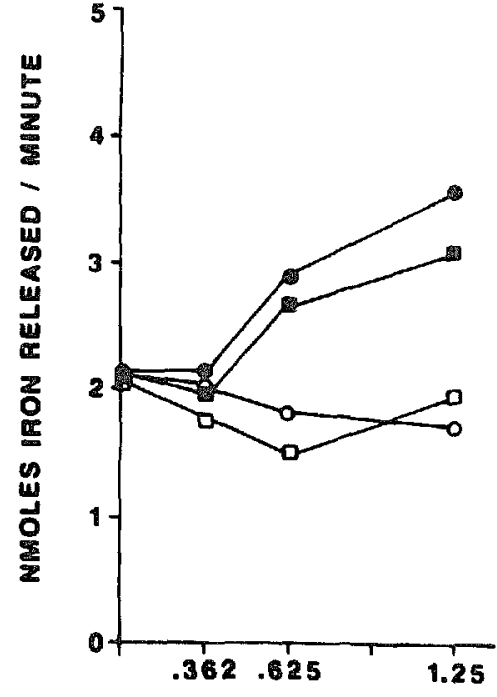

COMCENTAATHON [ $\mu \mathrm{H}$ ]

Fig. 2. Iron released ( $\mathrm{nmol} / \mathrm{min}$ ) from transferrin following addition of ATP of ADP $(0.16-1.25 \mu \mathrm{M})$ with or without incubation with apyrase $(4.9 \mathrm{U} / \mathrm{ml})$ at pH 5.45. ATP; O, ATP + apyrase; $\square$, ADP + apyrase.

quent assays were performed at $\mathrm{pH} 5.45$ to determine the effect of platelet conditioned media on the release of iron from transferrin.

The effect of adenine nucleotides, platelet sonicates or platelet conditioned media on the rate of iron released from transferrin

The effect of addition of adenine nucleotides, platelet sonicates or platelet conditioned media on the initial rate of iron released from transferrin at $\mathrm{pH} 5.45$ was determined. At pH 5.45, $2.40 \pm 0.13 \mathrm{nmol}$ of iron were released spontaneously from transferrin over the first minute of the reaction. Addition of ATP $(1.25 \mu \mathrm{M})$, platelet sonicates $\left(9.0 \cdot 10^{6}\right.$ platelet equivalents) or conditioned media from thrombin-stimulated platelets $\mathbf{( 9 . 0}$ $-10^{6}$ cell equivalents) significantly enhanced the rate of iron released from transferrin: $3.59 \pm 0.36,5.28 \pm 0.40$, and $5.89 \pm 0.25 \mathrm{nmol}$ iron released per min, respectively.

The effect of addition of varying concentrations of ATP and ADP on the rate of iron released from transferrin during the first minute of the reaction was determined (Fig. 2). Both ATP and ADP increased the rate of iron released from transferrin over a concentration range of 0.16 to $1.25 \mu \mathrm{M}$. At each concentration, ATP was more effective in potentiating iron release from transferrin than was ADP. Addition of either ATP or ADP that had been incubated with apyrase failed io enhance the spontaneous rate of iron released from transferrin at $\mathbf{p H} 5.45$.

The addition of varying amounts of conditioned media from both unstimulated and thrombin-stimulated platelets $\left(3.6 \cdot 10^{8}\right.$ platelets $\left./ \mathrm{ml}\right)$ enhanced the rate of 


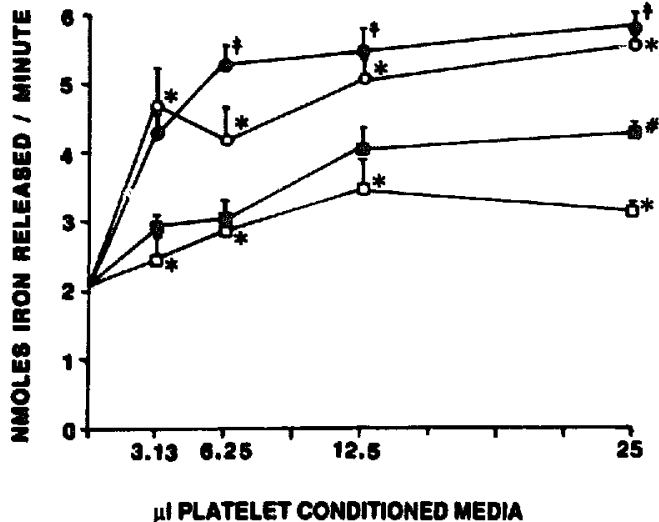

Fig. 3. Iron released (nmol/min) from transferrin following addition of conditioned media from unstimulated and thrombin-stimulated platelets $\left(0-9 \cdot 10^{6}\right.$ platelets) with or without incubation with apyrase at pH 5.45. The rate of iron released following addition of conditioned media from thrombin-stimulated platelets was compared to the rate following addition of conditioned media from unstimulated platelets; ( $\neq, P<0.05$ ). The rate of iron release following addition of conditioned media from unstimulated or thrombin-stimulated platelets was compared to the rate following addition of apyrase-treated conditioned media from comparatively stimulated platelets; ( $\#=P<$ 0.05). The rate of iron release following addition of apyrase-treated conditioned media from unstimulated or thrombin-stimulated platelets was compared to the spontaneous rate of iron released from transferrin; (* $=P<0.05)-$ conditioned media from thrombinstimulated platelets; $O$, conditioned media from thrombin-stimulated platelets + apyrase; , conditioned media from unstimulated platelets;

$\square$, conditioned media from unstimulated platelets + apyrase.

iron released from transferrin over basal levels at $\mathrm{pH}$ 5.45 (Fig. 3). In those experiments where greater than $3.13 \mu \mathrm{l}$ of platelet conditioned media was added, significantly more iron was released/min from thrombinstimulated platelets than from equivalent amounts of conditioned media from unstimulated platelets (Fig. 3). The relationship between the rate of iron released from transferrin during the first minute of the reaction and the volume of platelet conditioned media added was curvilinear, increasing as the volume of platelet conditioned media from either unstimulated or thrombin-

\section{TABLE I}

Ability of 'treated' conditioned media versus 'untreated' conditioned media from thrombin-stimulated platelets to facilitate the release of iron from transferrin

Conditioned media from thrombin-stimulated platelets was 'treated' as described in Methods. Results represent the mean of three separate experiments.

\begin{tabular}{lc}
\hline Treatment & $\begin{array}{l}\text { \% Recovery } \\
\text { of activity }\end{array}$ \\
\hline Platelet conditioned media & 100 \\
Filtrate (10000 molecular weight cutoff) & 105 \\
Heated conditioned media $\left(80^{\circ} \mathrm{C}, 30 \mathrm{~min}\right)$ & 97 \\
Conditioned media after freezing $\left(-20^{\circ} \mathrm{C}, 24 \mathrm{~h}\right)$ & 96 \\
Lipid phase (lipid extraction) & 11 \\
Aqueous phase (lipid extraction) & 106 \\
\hline
\end{tabular}

stimulated platelets was increased. Incubation of conditioned media from unstimulated and thrombin-stimulated platelets with apyrase resulted in a small decreased in the rate of iron released from transferrin (Fig. 3). The decrease in rate of iron released observed with apyrase was only significant when relatively large volumes of conditioned media from unstimulated cells $\left(9 \cdot 10^{6}\right.$ platelet equivalents) were added. The rate of iron released from transferrin following addition of apyrase-treated platelet conditioned media was significantly greater than the rate of iron released spontaneously at pH 5.45 (Fig. 3).

Using reverse phase HPLC methodologies, it was determined that $10^{8}$ platelets contained $7.55 \pm 3.10 \mathrm{nmol}$ of ATP and $9.62 \pm 1.53 \mathrm{nmol}$ of ADP, respectively. Similarly, $10^{8}$ platelets spontaneously secreted $1.31 \pm$ $0.45 \mathrm{nmol}$ ATP and $2.52 \pm 0.50 \mathrm{nmol}$ ADP, respec tively. Following stimulation of $10^{8}$ intact platelets with thrombin, the amount of ATP and ADP secreted by platelets was greatly enhanced $(4.30 \pm 0.58 \mathrm{nmol}$ ATP and $6.91 \pm 0.41 \mathrm{nmol}$ ADP). Following incubation with apyrase, the amount of ATP and ADP in platelet sonicates $\left(10^{8}\right.$ platelet equivalenis) was greatly diminished $(0.22 \pm 0.09 \mathrm{nmol}$ ATP and $0.01 \pm 0.01 \mathrm{nmol}$ ADP). Similarly, no detectable amount of ATP or ADP was measured in conditioned media from either unstimulated or thrombin-stimulated platelets following incubation with apyrase.

Characterization of the platelet-derived mediator(s) that promoted the release of iron from transferrin

Results of experiments to characterize the platelet derived mediator(s) that facilitated the release of iron from transferrin are shown in Table 1 . These results suggest the mediator(s) has a low molecular weight $(<10000)$, is stable at extreme temperatures $\left(80^{\circ} \mathrm{C}, 30\right.$ min; $-20^{\circ} \mathrm{C}, 24 \mathrm{~h}$ ) and is not lipid extractable.

\section{Discussion}

Previously published reports indicate that the release of iron from transferrin is dependent on the hydrogen ion concentration $[3,7,15,16]$. These studies suggest that the release of iron may be accelerated at low $\mathrm{pH}$ values because of: (i) protonation of bicarbonate with subsequent loss of the anion and labilization of the iron, (ii) protonation of functional groups on transferrin resulting in alteration of charge of the molecule or the conformational stability of the ternary iron-transferrinbicarbonate complex, (iii) alteration in the degree of protonation of the attacking chelate [15]. Our results extend these observations showing that the addition of human platelet sonicates or conditioned media from unstimulated or thrombin-stimulated platelets enhanced the initial rate of iron released from transferrin at $\mathrm{pH}$ values of 6.2 or less. 
Given that addition of conditioned media from unstimulated or thrombin-stimulated platelets enhanced the rate of iron release from transferrin at pH 5.45 (Fig. 3 ), we determined if platelet conditioned media facilitated the release of iron by adenine nucleotide-dependent or -independent mechanisms. Previous studies have shown that adenine nucleotides will facilitate the release of iron from transferrin [7]. Therefore, we quantitated the amount of adenine nucleotides (ATP, ADP) present in conditioned media from platelets. In agreement with previously published reports [10], we observed that detectable amounts of these nucleotides were secreted spontaneously from intact human platelets after in vitro purification and the concentration of nucleotides secreted by intact platelets was greatly enhanced following thrombin stimulation. However, the rate of iron released after addition of $0.12 \mu \mathrm{M}$ ATP and $0.23 \mu \mathrm{M}$ ADP (i.e. equivalent to that found in conditioned media from $9 \times 10^{6}$ unstimulated platelets) would be approximately $2.1 \mathrm{nmol} / \mathrm{min}$ (Fig. 2) (assuming the eifect of these two agents is additive) while the rate observed following addition of cotditioned media from $9 \cdot 10^{6}$ unstimulated platelets was $4.32 \pm 0.08 \mathrm{nmol} / \mathrm{min}$ (Fig. 3 ). Similarly, the rate of iron release following addition of $0.39 \mu \mathrm{M}$ ATP and $0.63 \mu \mathrm{M}$ ADP (i.e. equivalent to concentrations present in conditioned media from $9 \cdot 10^{6}$ thrombin-stimulated platelets) would be approx. 2.7 $\mathrm{nmol} / \mathrm{min}$ (assuming the effect of ATP and ADP is additive) while the observed rate following addition of conditioned media from thrombin-stimulated platelets $\left(9 \cdot 10^{6}\right)$ was $5.89 \pm 0.25 \mathrm{nmol} / \mathrm{min}$. These results suggest that there are mediators released from unstimulated and thrombin-stimulated platelets that facilitate the release of iron from transferrin by adenine nucleotide-independent mechanisms.

To further assess the contribution of platelet-derived adenine nucleotides in facilitating the rate of iron release from transferrin, we eliminated ATP and ADP from platelet conditioned media by incubating samples with apyrase. The absence of the adenine nucleotides in apyrase-treated conditioned media was confirmed by HPLC. Addition of apyrase to purified ATP or ADP completely eliminated any enhancement in the rate of iron release from transferrin over basal levels. In contrast, pre-incubation of conditioned media from either unstimulated or stimulated platelets with apyrase did not significantly alter their ability to enhance the rate of iron released over basal levels at most volumes of platelet conditioned media assayed. This indicates that the release of iron from transferrin by mediators present in conditioned media from unstimulated or stimulated platelets occurs primarily by adenine nucleotide-independent mechanisms.

Previous investigators have proposed an 'interlocking site model' to illustrate the binding of iron to transferrin in the Fe-transferrin-bicarbonate complex [17]. They suggest that in the ternary iron-bicarbonate-transferrin complex, bicarbonate is bound to the cationic protein side groups immediately in the vicinity of the metal binding site and also provides an oxygen as a direct ligand to $\mathrm{Fe}^{3+}[17]$. Removal of iron from diferric transferrin is a multistep reaction and may involve one or more of the following factors: (i) chelation, (ii) reduction, (iii) weakening of the bicarbonate iron linkage, (iv) disruption of the amino acid residues in the $\mathrm{Fe}^{3+}$ binding site, (v) conformational changes [18]. Results of recent studies suggest that a conformational change in the ferric-transferrin-bicarbonate molecule is the rate-limiting step for iron removal by nonreducing chelators [19-22]. The general scheme for iron removal by this mechanism (i.e. the Bates mechanism) involves a conformational change in the stable diferric transferrin from the 'closed' configuration (presumably in this configuration the iron is buried within the protein) to the 'open apotransferrin-like' configuration (presumably where the metal binding sites are accessible) [21-23]. We suspect that addition of mediators present in platelet conditioned media may also facilitate conformational changes in the iron-transferrin-bicarbonate complex thus enhancing the labilization of iron.

In summary, both unstimulated and thrombinstimulated platelets release mediators that facilitate the release of iron from transferrin in an acidic environment. Although the platelet-derived adenine nucleotides ATP and ADP potentiate the exchange of bicarbonate and thereby promote the labilization of iron, it is apparent that additional adenine nucleotide-independent mediators are released from platelets that also enhance the release of iron from transferrin. These mediators, like ATP and ADP, are released spontaneously from human platelets in vitro and their release is enhanced following thrombin-induced platelet aggregation. Since iron has been shown to promote oxidant-dependent phagocytic cell cytotoxicity and tissue injury both in vitro and in vivo $[24,25]$, platelets may function to promote tissue injury at sites of inflammation secondary to their ability to enhance the release of iron from transferrin. Released iron could promote propagation of lipid peroxidation reactions or hydroxyl radical formation following reaction with phagocytic cell hydrogen peroxide. The restriction of this effect to conditions of acid $\mathrm{pH}$ limits its pathophysiologic role to disease processes that result in a significant decrease in tissue $\mathrm{pH}$ or within the microenvironment of activated phagocytic cells where the $\mathrm{pH}$ at the cell surface is less than $6.0[26]$.

\section{Acknowledgements}

This work was supported in part by National Institute of Health Grants HL-32024, HL-28737, and RR-07008 and a Grant-in-Aid from the American Heart 
Association. Dr. Sem Phan is a recipient of an Established Investigatorship from the American Heart Association.

\section{References}

1 Minotti, G. and Aust, S.D. (1987) J. Biol. Chem. 262, 1098-1104. 2 Girotti, A.W. (1985) J. Free Radicals Biol. Med. 1, 87-95.

3 Carver, F.J. and Frieden, E. (1978) Biochemistry 17, 167-172.

4 Kojima, N. and Bates, G.W. (1979) J. Biol. Chem. 254, 884i-8854.

5 Harris, D.C., Rinehart, A.L., Hereld, D., Schwartz, R.W., Burke, F.P. and Salvador, A.P. (1985) Biochim. Biophys. Acta 838, 295-301.

6 Huebers, H.A. and Finch, C.A. (1987) Physiol. Rev. 67, 520-528.

7 Morgan, E.H. (1979) Biochim. Biophys. Acta 580, 312-326.

8 Egyed. A. (1975) Biochim. Biophys. Acta 411, 349-356.

9 Rogers, T.B., Feeney, R.E. and Meares, D.F. (1977) J. Biol. Chem. 252, 8108-8112.

10 Karpatkin, S. and Langer. M. (1968) J. Clin. Invest. 47, 2158-2168.

11 Leung, L. and Nachman, R. (1986) Annu. Rev. Med. 37, 179-186.

12 Lorenzet, R., Niemetz, J., Marcus, A.J. and Broekman, M.J. (1986) J. Clin. Invest. 78, 418-423.

13 Rao, G.H.R., Peller, J.D. and White, J.C. (1981) J. Chromatogr. 226, 466-470.
14 Bligh, E.G. and Dyer, W.J. (1959) Can. J. Biochem. Physiol. 37, 911-917.

15 Baldwin, D.A., De Sousa, D.M.R. and Von Wandruszka, R.M.A. (1982) Biochim. Biophys. Acta 719, 140-146.

16 Thompson, C.P., Grady, J.K. and Chasteen, N.D. (1986) J. Biol. Chem. 261, 13128-13134.

17 Harris, W.R. (1985) Biochemistry 24, 7412-7418.

18 Graham, G.A. and Bates, G.W. (1981) in Proteins and Iron Metabolism (Brown, E.B., Aisen, P., Fielding, J. and Crichton, R.R., eds.), pp. 227-236, Grune and Stratton, New York.

19 Cowart, R.E., Swope, S., Loh, T.T., Chasteen, N.D. and Bates, G.W. (1986) J. Biol. Chem. 261, 4607-4614.

20 Cowart, R.E., Kojima, N. and Bates, G.W. (1982) J. Biol. Chem. 257, 7560-7565.

21 Ketchmar, S.A. and Raymond, K.N. (1986) J. Am. Chem. Soc. 108, 6212-6218.

22 Konopka, K., Bindereif, A. and Neilands, J.B. (1982) Biochemistry 21, 6503-6508.

23 Bates, G.W. (1982) in The Biochemistry and Physiology of Iron (Saltman, P. and Hegenauer, J., eds.), pp. 3-18, Elsevier/NorthHolland, Amsterdam.

24 Fantone, J.C. and Ward, P.A. (1982) Am. J. Pathol. 107, 395-418. 25 Weiss, S.J. and LoBuglio, A.F. (1982) Lab. Invest. 47, 5-18.

26 Etherington, D.J., Pugh, D. and Silver, I.A. (1981) Acta Biol. Med. Germ. 40, 1625-1636. 